\title{
Left Ventricular Outflow Tract Obstruction Defined by Active Three-Dimensional Echocardiography Using Rotational Transthoracic Acquisition
}

\author{
DEREK A. FYFE, M.D., PH.D., ACHI LUDOMIRSKY, M.D., * \\ SATINDER SANDHU, M.D., ,** PRADIP K. DHAR, M.D., \\ MICHAEL SILBERBACH, M.D.**, and DAVID J. SAHN, M.D.** \\ South Carolina Children's Heart Center, Medical University of S.C., Charleston, South Carolina; \\ *Department of Pediatrics (Cardiology), University of Michigan, Ann Arbor, Michigan; and \\ **Center for Congenital Heart Disease, Oregon Health Sciences University, Portland, Oregon
}

A rotational data acquisition system was used to create three-dimensional images from thoracic and subxiphoid echocardiographic windows in children with various types of subaortic stenosis. Thirteen patients, ranging in age from 2 days to 17 years, were examined. Subaortic obstruction was caused by a discrete fibrous ridge in six patients, hypertrophic cardiomyopathy in two patients, subaortic tunnel in two patients, and septal malalignment, restrictive VSD, and abnormal suture placement each in one patient. Unique views could be obtained equivalent to surgical or autopsy dissections, and allowed more complete understanding of morphology than conventional imaging techniques. (ECHOCARDIOGRAPHY, Volume 11, November 1994)

three-dimensional echocardiography, ventricular outflow obstruction

Subaortic stenosis may occur due to a variety of structural malformations. ${ }^{1}$ An accurate morphological understanding is critical to planning and performing surgical treatment. Up until recently, two-dimensional echocardiography or angiography were the preferred imaging techniques. ${ }^{24}$

Three-dimensional imaging has now become feasible for echocardiographic use.-7 Three-dimensional echocardiography of structural anomalies of the subaortic left ventricular outflow tract has recently been reported. ${ }^{8}$ The technique employed utilized a transducer designed for transesophageal use in which parallel echo scans or "slices" were sequentially obtained across the chest, as the transducer was mechanically withdrawn within a carriage to obtain a dataset. ${ }^{9}$ Recently a rotational acquisition system has been developed in which a standard, commercially

Address for correspondence and reprints: Derek A. Fyfe, M.D., Ph.D., The Children's Heart Center, 2040 Ridgewood Dr. NE, Atlanta, GA 30322. Fax: 404-248-5110. available, transthoracic echo transducer is placed in a rotating mechanical motorized holder, and sequential scans are obtained. Slices of these "propeller-like" scans are then analyzed, synthesized, and displayed. This technique has significant technical advantages over other techniques, as only a single echocardiographic window is required for all image acquisition. To evaluate the diagnostic utility of rotational acquisition, we performed three-dimensional imaging in a series of patients with a spectrum of obstructive lesions of the left ventricular outflow tract. Unique views obtained with three-dimensional reconstruction simulated those only obtainable during surgical exposure or anatomical dissection.

\section{Methods}

A prototype dynamic three-dimensional acquisition and processing system (Tomtec, Broomfield, CO, USA) was used, linked to commercially available Interspec Apogee (Ambler, 
PA, USA) or CX 200 echocardiography systems already in clinical use.

Either 7.5-, 5-, or 3.5-MHz probes were used, mounted in a cylindrical, motorized transducer holder. After a clear transthoracic or subxiphoid echo window, which included the region of interest, was located, the transducer was locked in this position with the use of a mounting arm. The probes were mechanically rotated $180^{\circ}$ in $1^{\circ}-2^{\circ}$ increments by the stepper motor during data acquisition. The data collection was gated for both cardiac cycle and respiration using standard ECG connections. The image acquisition was monitored on the echocardiographic machine during rotation. Following image acquisition, the computer was disconnected from the echo machine and offline processing was performed by selection of critical regions of interest. Datasets were oriented and then cut in differing planes to obtain the optimal imaging orientation for display. Surface and volume rendering was then performed.

\section{Patient Preparation}

Infants and children were examined at the conclusion of standard echocardiography examinations, which were being performed as a clinical service. In some cases, sedation had been administered for the clinical exam, and patient monitoring adhered to established guidelines for pediatric sedation. ${ }^{10}$ Data collection usually required about 5-10 minutes to set the transducer holding arm in position, optimize the image, and calibrate the system. The actual scan time to acquire a complete dataset was 1-2 minutes for each echocardiographic window once the views were optimized. The patients were then dismissed from the laboratory and image analysis performed off line. Reconstruction to the first image following orientation and selection of thresholds, choice of pixel density, and application of surface shading techniques could be achieved in as short a time as $\mathbf{1 0}$ minutes. If higher pixel density was selected, computation was slightly longer.

\section{Examination Windows}

Standard subxiphoid, apical, and parasternal transthoracic echocardiographic windows were used for image acquisition, depending on which window provided the best access to imaging the relevant anatomy.

\section{Results}

Thirteen patients with subaortic obstruction were studied ranging in age from 2 days to 17 (median 1) years of age (Table I). Five patients required sedation for study.

TABLE I

\begin{tabular}{|c|c|c|c|c|}
\hline Patient & Age & $\begin{array}{c}\text { Weight } \\
\text { kg }\end{array}$ & Diagnosis & $\begin{array}{l}\text { 3-D Echo } \\
\text { Window }\end{array}$ \\
\hline 1 & 3 months & 4 & discrete membranous & left parasternal \\
\hline 2 & 4 months & 4.2 & discrete membranous & left parasternal \\
\hline 3 & 6 months & 6 & IHSS & right parasternal \\
\hline 4 & 2 years & 15 & tunnel & left parasternal \\
\hline 5 & 4 years & 25 & IHSS & left parasternal \\
\hline 6 & $41 / 2$ years & 30 & discrete membranous & left parasternal \\
\hline 7 & 1 year & 8 & recurrent discrete & subxiphoid \\
\hline 8 & 4 years & 17 & tunnel & left parasternal \\
\hline 9 & 2 days & 3.0 & malaligned septum, IAA & subxiphoid \\
\hline 10 & 4 months & 7.2 & DORV, restrictive VSD & subxiphoid \\
\hline 11 & 6 years & 28 & discrete membranous & subxiphoid \\
\hline 12 & 8 years & 29 & discrete membranous & subxiphoid \\
\hline 13 & 17 years & 68 & discrete membranous & apical \\
\hline
\end{tabular}

IHSS = idiopathic hypertrophic subaortic stenosis; IAA = interrupted aortic arch; DORV = double outlet right ventricle; $\mathrm{VSD}=$ ventricular septal defect. 


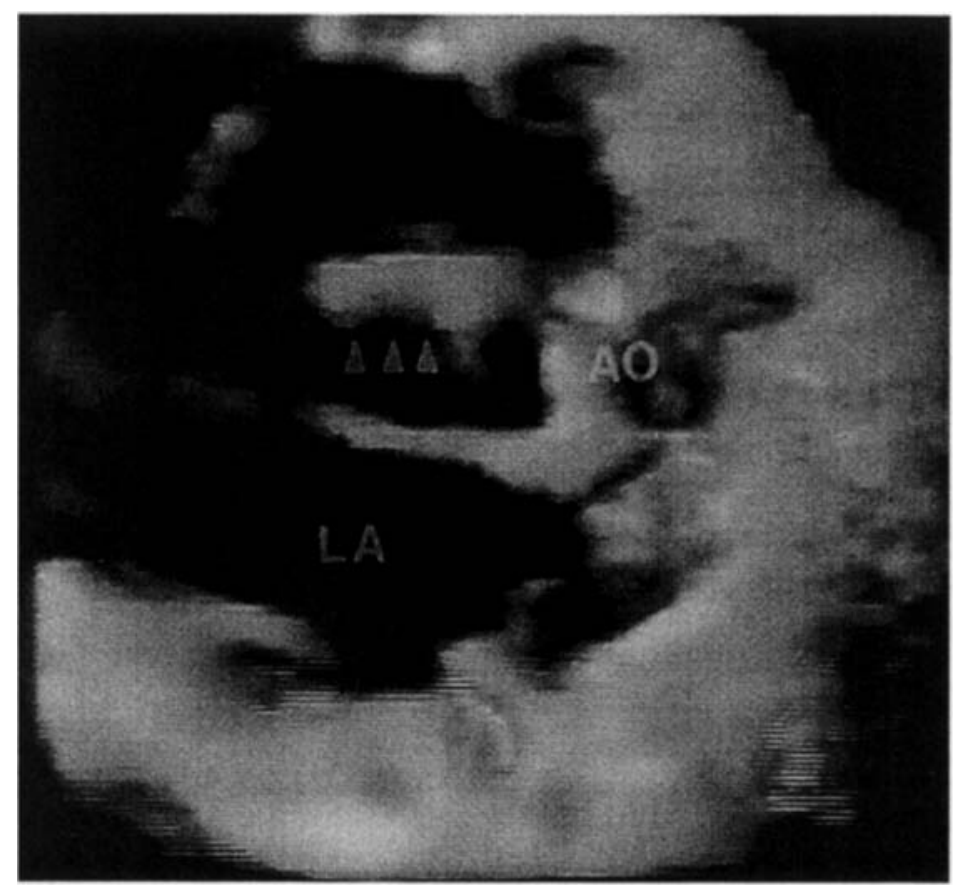

Figure 1A. Three-dimensional reconstruction showing the full extent of a tunnel-like abnormality (arrows) shown in a long-axis cut.

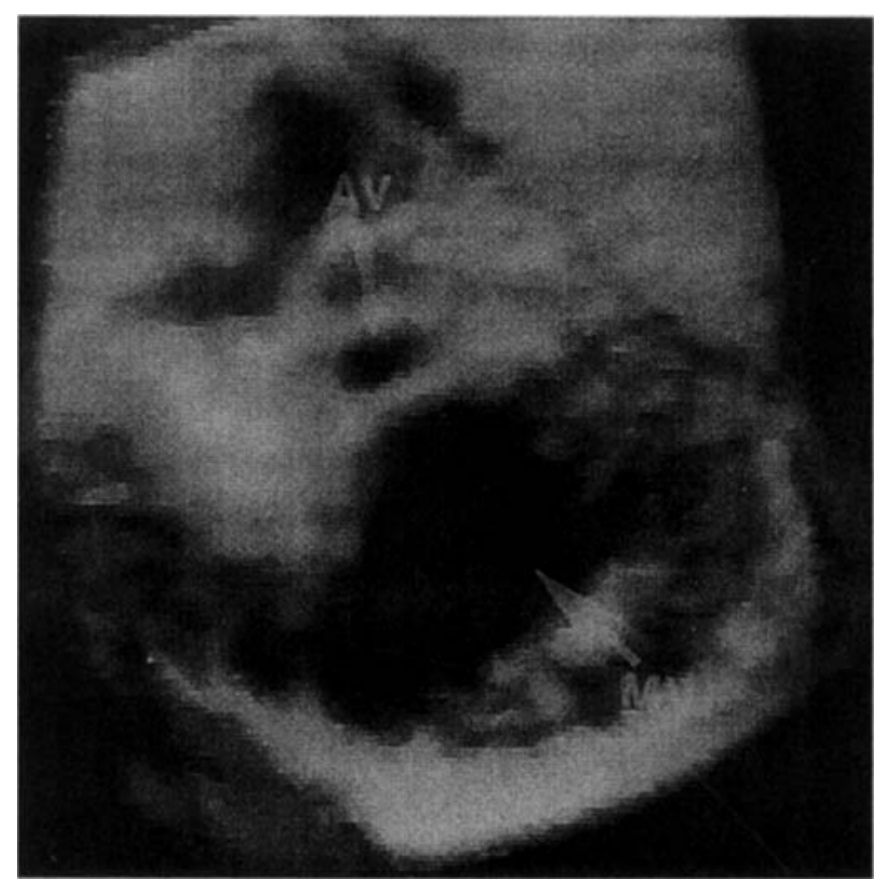

Figure 1B. Shows the subaortic tunnel in cross-section viewed from within the ventricle looking up at the aortic valve (AV). MV = mitral valve. 


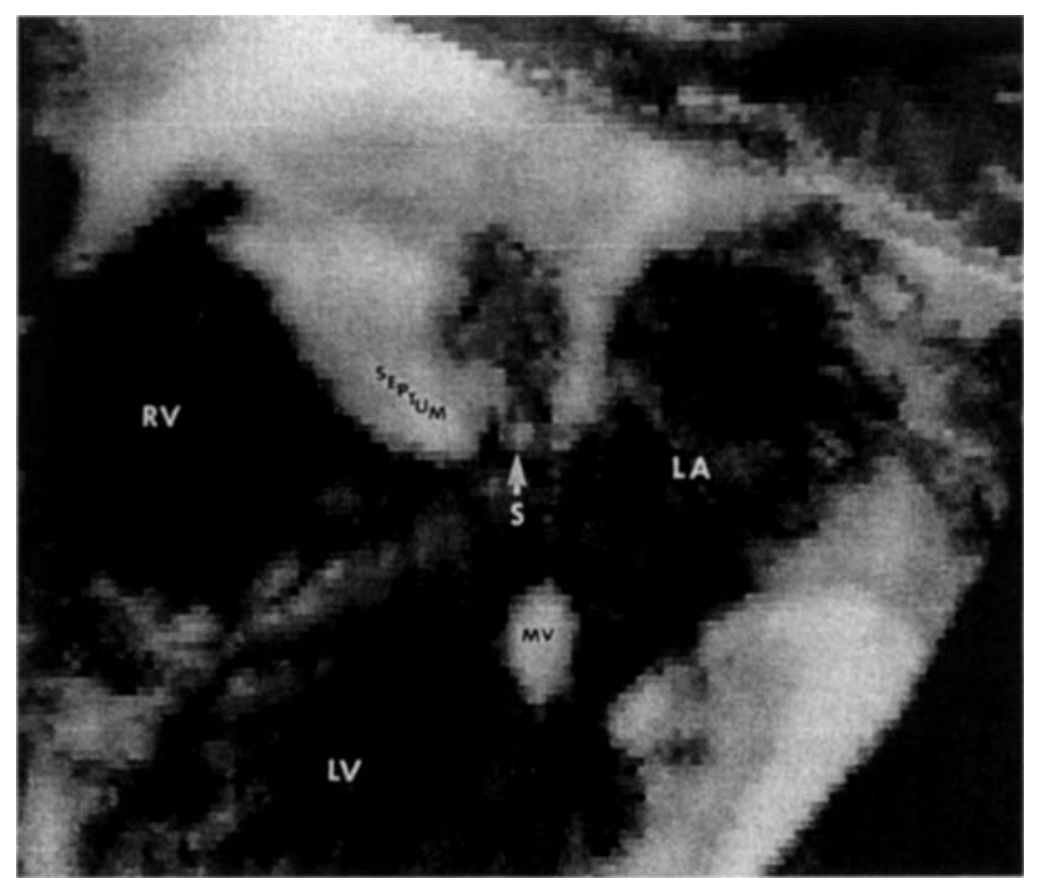

Figure 2. Three-dimensional view of subaortic septal malalignment from subxiphoid window. The malaligned subaortic septum can be seen as the obstructive lesion (S). The right ventricle $(R V)$ is seen through the VSD, which is seen below and its septal margins spatially defined. VSD $=V S D ; L A=$ left atrium; $L V=$ left ventricle; $M V=$ mitral valve.

\section{Subaortic Obstruction}

Tunnel subaortic stenosis was examined in two cases (Fig. 1). The tubular nature of the obstruction is well displayed using views in which the outflow tract is cut longitudinally from apex to base and viewed from an anterior location. The diffuse distribution of the obstructive tissue is seen with circumferential narrowing due to the muscular obstruction, extending from aortic to mitral leaflets.

\section{Subaortic Septal Malalignment}

A patient of 2 days of age, weighing 3.0 kilograms, with normally related great vessels, ventricular septal defect (VSD), and interruption of the aortic arch type B is shown. The left ventricular (LV) outflow views are shown (Fig. 2 ). The dataset was cut from apex to base alongside the ventricular septum. The discontinuity and malalignment of the subaortic ventricular septum is clearly seen as is the crest of the large VSD. A second view was obtained in which the VSD and subaortic septum are examined en face, as if the observer is standing on the inside free wall of the left ventricle looking toward the septum (Fig. 3). The size and relations of the VSD are appreciated easily, and the posterior and anterior extension of subaortic septum is well seen.

\section{Iatragenic Septal Outflow Obstruction}

Following closure of a VSD, which was inadvertently created during resection of a subaortic membrane, a large partially obstructive pledgetted suture was placed in the left side of the septum in the outflow tract. The discrete nature of this lesion is clearly appreciable looking from the apex of the heart into the outflow tract toward the aortic valve (Fig. 4).

\section{Restrictive Ventricular Septal Defect}

Left ventricular outflow obstruction after repair of double outlet right ventricle was also 


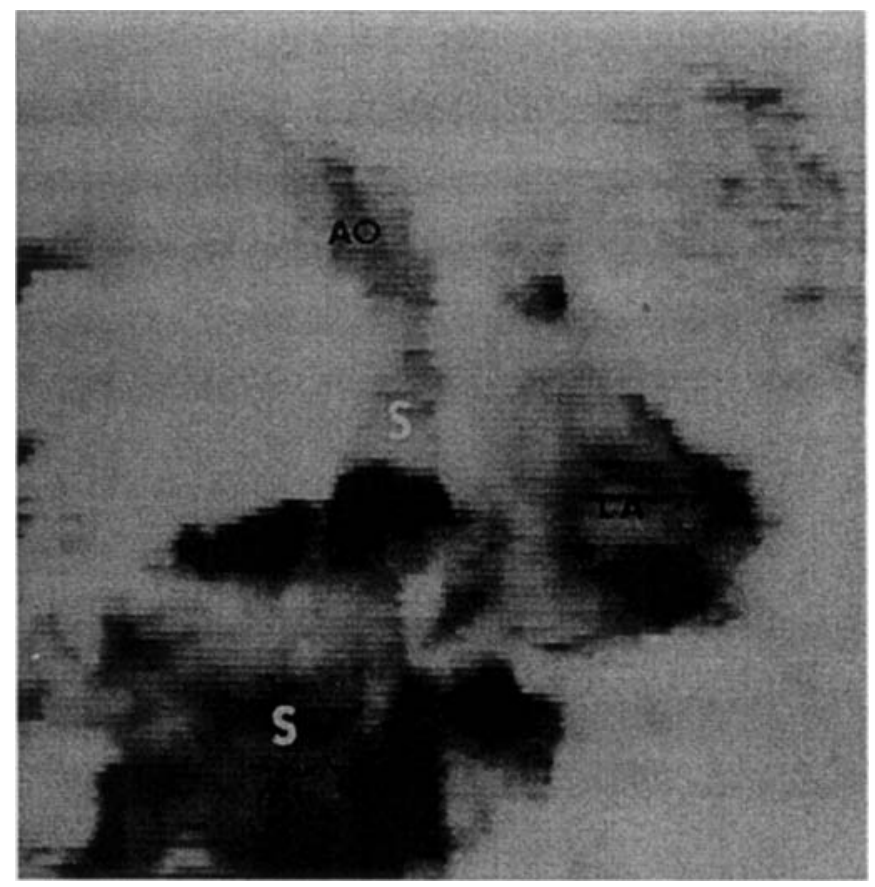

Figure 3. View of the LVOT in a child (also seen in Fig. 2) with subaortic septal malalignment looking en face at the septum (S) above and below the large muscular VSD viewed from $L V$ freewall. $L V O T=$ left ventricular outflow tract; $L V=$ left ventricle.

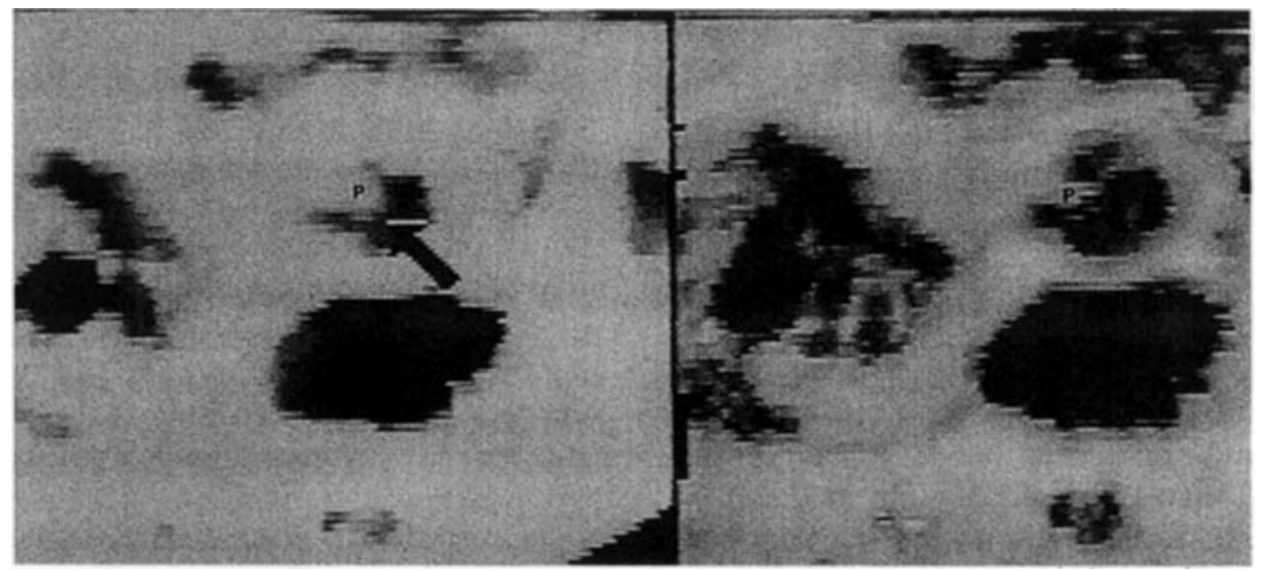

Figure 4. Three-dimensional view from LV looking up at a pledget-suture protruding into the outflow tract below the aortic valve. Left and right panels shows alternative surface shading techniques. $L V=$ left ventricle. 


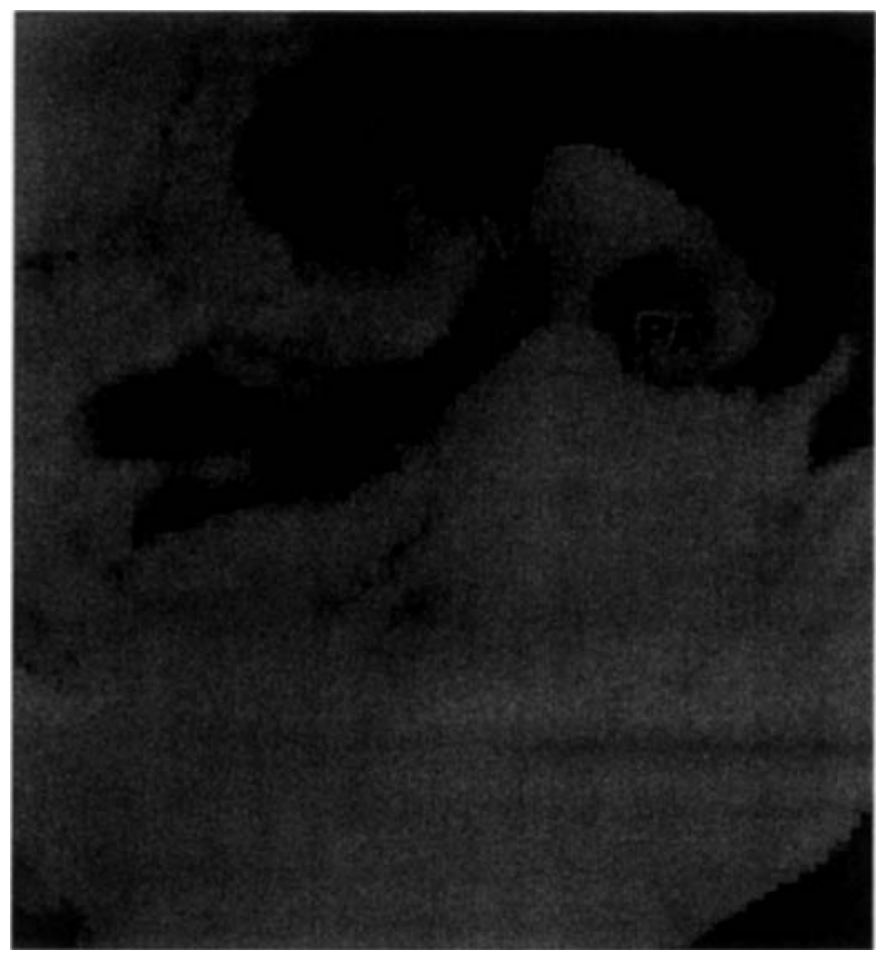

Figure 5. Long-axis views of a restrictive VSD and a portion of the left ventricle to aortic tunnel after double outlet right ventricle repair. The RVOT connection to the pulmonary artery is not seen. VSD = ventricular septal defect; $R$ VOT $=$ right ventricular outflow tract.

defined in one patient (Fig. 5). The outflow obstruction was due to a restrictive VSD and the relation to the great arteries was well appreciated.

\section{Hypertrophic Cardiomyopathy}

Two patients with idiopathic subaortic stenosis were studied showing the dynamically changing septal thickening as the cause of obstruction.

\section{Discrete Fibrous Subaortic Stenosis}

This was the most frequent lesion examined (six patients). The complete extent of the subaortic membrane is seen crossing the outflow tract viewed in long axis (Figs. 6 and 7). This can be best appreciated from the ventricular side of the outflow tract looking toward the aortic valve. In a surgeon's eye view through the aortic valve, the position and severity of obstruction can likewise be clearly defined (Fig. 8).

\section{Discussion}

Subaortic stenosis may occur due to a spectrum of structural abnormalities..$^{1-4}$ Two-dimensional echocardiography or contrast angiography have usually provided the diagnostic imaging, however, in both techniques, the cardiologist must create a mental three-dimensional image, and may need also to try to convey this structural information to a surgeon.

Three-dimensional echocardiography in this study allowed a more lifelike spatial representation of the anatomical details of the various morphological causes of subaortic obstruction, which can help in understanding and in planning surgery. Long and short axial alignments through cut surfaces provided unique images in all cases.

The rotational acquisition system utilized in this study is a new technique that avoids many previous problems with image location and registration, as the probe gathers the entire dataset from one location. Previous reports have used 


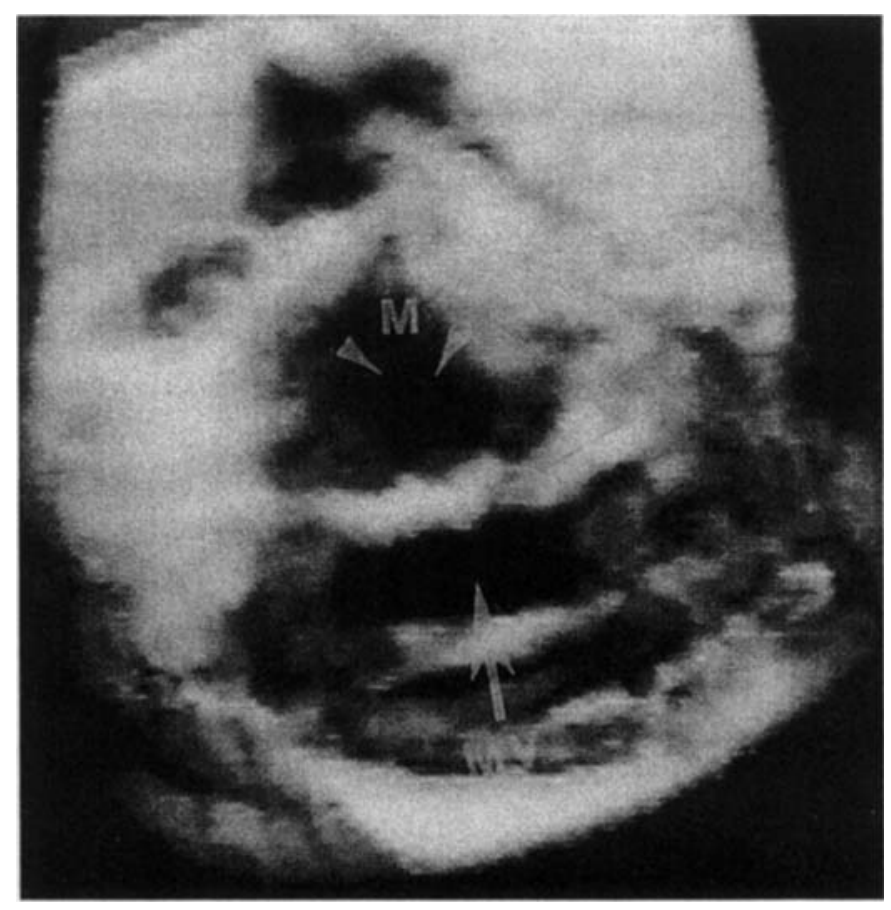

Figure 6. Three-dimensional view in a unique projection, looking from the inside of the left ventricle into the outflow tract. The membrane $(M)$ is protruding into the outflow tract during systole. $M V=$ mitral valve.

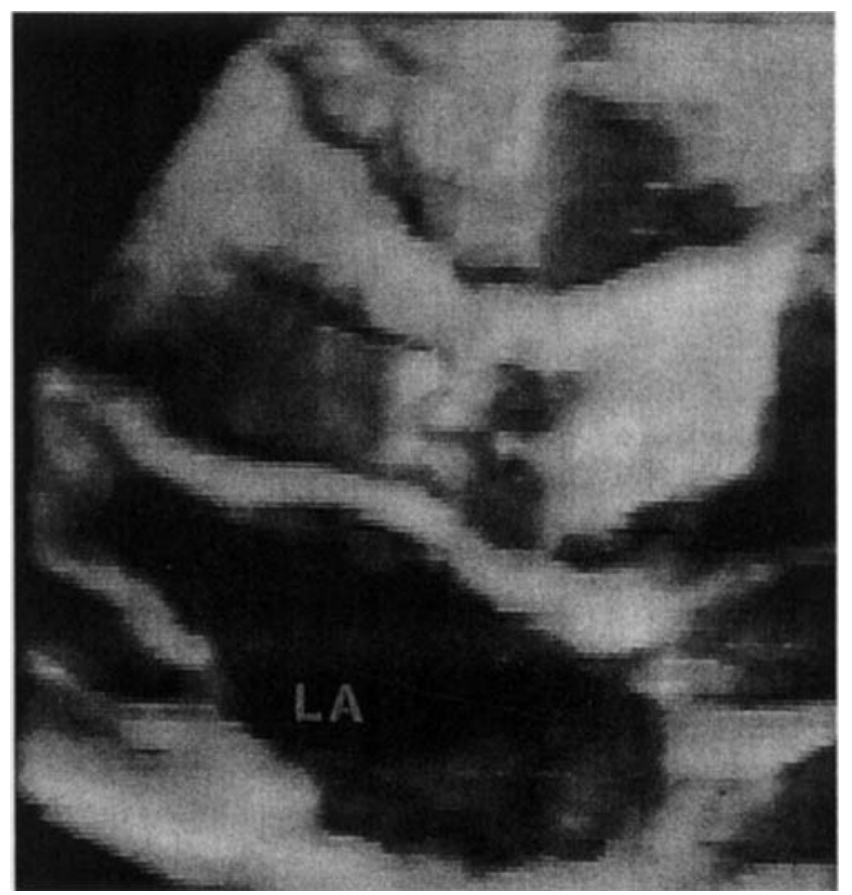

Figure 7. Three-dimensional view of subaortic fibrous membrane in a similar orientation to a parasternal long-axis scan. An incomplete membrane (arrow) is seen below the aortic valve. The extent of the membranous obstruction is well seen. $A O=$ aorta; $L A=$ left atrium. 


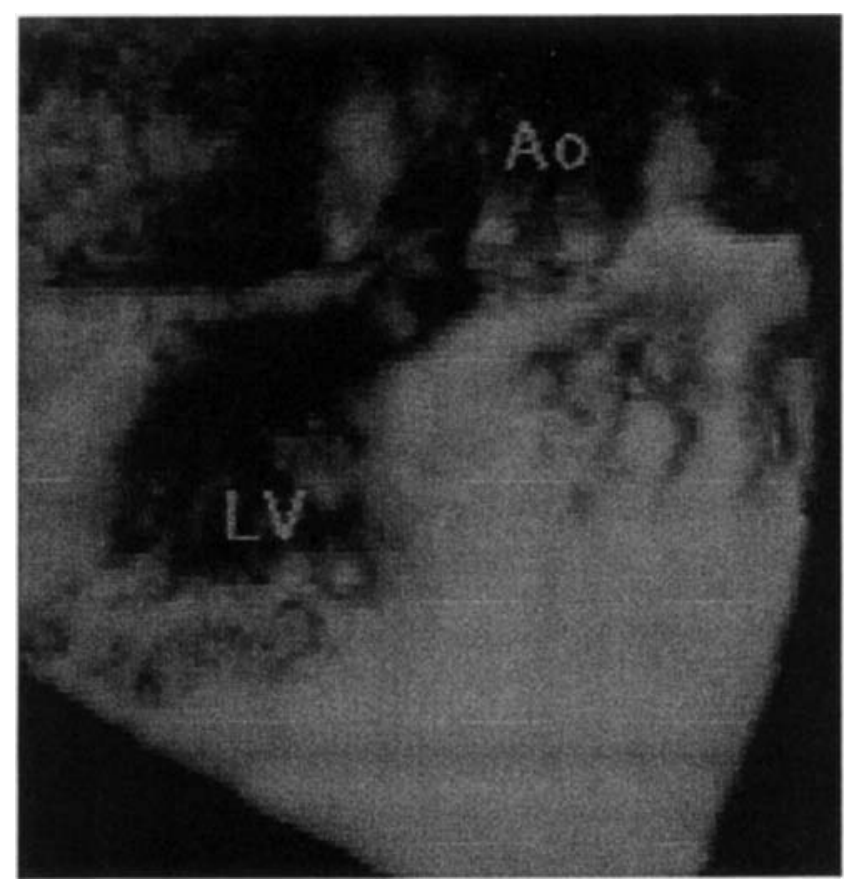

Figure 8. Long-axis views of a discrete membrane at an unusually long distance of the valve.

parallel slicing techniques, which require the imaging transducer to slide over the chest, limiting to some extent the windows for data acquisition. ${ }^{5}$ The rotational acquisition system has the advantage that a single window can optimally provide a site for data acquisition which is not limited by ribs, etc., as a sliding probe in a carriage may be. Subxiphoid windows are most easily utilized with little or no perception of movement of the probe by the patient. Subxiphoid windows may be potentially less likely to have problems of probe motion due to respiratory excursion of chest wall. Also, there is the potential for high resolution due to overlap of scan planes at the center of the dataset. Apical positions may be more difficult due to the chest wall movement interferring with the ability to hold the transducer in a stable position while maintaining constant contact. However, in this study, when stable contact was obtained, excellent clarity of images was achieved.

The disadvantages of the rotational acquisition method are that it may have limited resolution in the far field due to the increasing separation of scan planes as the distance from center of dataset increases. This problem can be reduced or avoided by centering the primary region of interest in the screen during data acquisition. Rotational artifacts also occur due to the $180^{\circ}$ scan set needed to maintain a perfect center of rotation during the acquisition in order to align data from opposite sides of the imaging fan following rotation.

Currently, Doppler flow can be visualized, but only in a gray scale image superimposed upon the echocardiographic image. More research into quantitative aspects of color flow and three-dimensional reconstruction is necessary before clinical information can be assessed.

In summary, unique new views of the heart in this study of the left ventricular outflow tract, equivalent to those which could be seen only by surgical or autopsy exposure, were accomplished. Lifelike views allow full appreciation of surgically important structures in real time. These "surgical" views have surfaces and, therefore, are unlike the shadows obtained by catheterization or two-dimensional echocardiography. The potential for image manipulation in virtual reality creates the possibility for experimental "pseudo"- 
surgical computer modeling prior to the actual performance of definitive surgical procedures, and may allow an accurate analysis of the likely surgical result.

\section{References}

1. Edwards JE: Pathology of left ventricular outflow tract obstruction. Circulation 1965;32:586-599.

2. Kandah T, Kimball TR, Daniels SR, et al: When is echocardiography unreliable in patients undergoing catheterization for pediatric cardiovascular disease? J Am Soc Echo 1991;4:51-56.

3. Wilcox WD, Seward JB, Hagler DJ, et al: Discrete subaortic stenosis: Two-dimensional echocardiographic features with angiographic and surgical correlation. Mayo Clin Proc 1980;55:425-432.

4. Vogel M, Freedom RM, Brand A, et al: Ventricular septal. defect and subaortic stenosis: An analysis of 41 patients. Am $J$ Cardiol 1983;52:1258-1263.

5. Sheikh KH, Smith SW, von Ramm O, et al: Real-time, three-dimensional echocardiography: Feasibility and initial use. Echocardio- graphy 1991;8:119-125.

6. King DL, Harrison MR, King DL Jr, et al: Improved reproducibility of left atrial and left ventricular measurements by guided threedimensional echocardiography. JACC 1992; 20:1238-1245.

7. Pandian NG, Nanda NC, Schwartz SL, et al: Three-dimensional and four-dimensional transesophageal echocardiographic imaging of the heart and aorta in humans using a computed tomographic imaging probe. Echocardiography 1992;9:677-687.

8. Vogel M, Lösch S, Bühlmeyer K: The application of transthoracic dynamic three-dimensional echocardiography by computer-controlled parallel slicing in patients with fixed subaortic obstruction. Cardiol Young 1994;4:714.

9. Wollschläger $\mathrm{H}$, Zeiher AM, Klein $\mathrm{HP}$, et al: Transesophageal echo computer tomography: A new method for dynamic 3-D imaging of the heart (abstract). Circulation 1989;80:II-569.

10. Committee on Drugs: Guidelines for monitoring and management of pediatric patients during and after sedation for diagnostic and therapeutic procedures. Pediatrics 1992;89:11101115. 